Selim S

\title{
Department of Endocrinology, Bangabandhu Sheikh Mujib Medical University, Dhaka, Bangladesh
}

Background: Currently, it is known that the entire gastrointestinal tract is affected by DM, from the mouth to the anorectal region, and dysfunction of this system contributes to the morbidity of this disease and worsens the quality of life of diabetic individuals. Thus, there is need of achieving better understanding of the gastrointestinal symptoms in patients with DM and joining efforts of pediatricians and pediatric endocrinologists and gastroenterologists for the proper management of patients. So, the study was designed to explore types of GI disorders and their covariates in a group of type 2 DM subjects with more than 10 years of diabetes.

Design and Methods: A cross-sectional analytic study was conducted among on adult type 2 diabetes patients with duration of diabetes at least 10 years. The type 2 diabetes patients attending out-patient departments of diabetes care centers of Dhaka city Bangladesh, were interviewed by trained research physicians after obtaining informed consent form signed. They collected sociodemographic data by face to face interview and diabetes related data from the 'Guide book'. Rome IV diagnostic questionnaire for the adult was utilized for detection of diabetic Gastropathy. In total 3893 respondents were included in the study. Maintaining all the safety precaution, blood sample was collected glycemic status, liver function, kidney function and basic defects of diabetes through homeostasis model assessment.

Results: The characteristics of the study groups are shown in table 1 which shows the distribution of the diabetic patient by sociodemographic characteristics and presence of gastroparesis. The median age was 52 yrs in GID group and around 54 in NGID. No specific age differential is evident in two groups ( $P>0.05)$. Regarding sex distribution in male suffers more GID than female $(P<0.05)$. Although the disease is more prevalent among urban residents the difference is not statistically significant $(P>0.05)$.

\begin{tabular}{|c|c|c|c|}
\hline Variables & $\operatorname{NGID}(n=362)$ & $\operatorname{GID}(n=3531)$ & Test statistics \\
\hline \multicolumn{4}{|l|}{ Age } \\
\hline $\begin{array}{l}\text { median } \\
\text { Range }\end{array}$ & $\begin{array}{c}56.0 \\
(29.0-78.0)\end{array}$ & $\begin{array}{c}53.0 \\
(26.0-85.0)\end{array}$ & $\mathrm{z}=1.54, \mathrm{P}=.125$ \\
\hline \multicolumn{4}{|l|}{ Gender } \\
\hline Male & $142(39.3 \%)$ & 2094 (59.3\%) & $x^{2}=7.4, p=.025$ \\
\hline \multicolumn{4}{|l|}{ Residence } \\
\hline Urban & 207 (57.1\%) & 221 (61.2\%) & \multirow{2}{*}{$x^{2}=.173, p=.41$} \\
\hline Rural & 155 (42.9\%) & 104 (38.8\%) & \\
\hline
\end{tabular}

Different number of GI disorders in the study subjects

Figure 1 shows the data of different disorder groups in the study subjects. Among 301 subjects the percentage of no disorder was about $9.3 \%$, single disorder was about $40.9 \%$, double disorders were about $29.6 \%$, Triple disorders were about $11.3 \%$ and more than triple disorders were about $9 \%$.

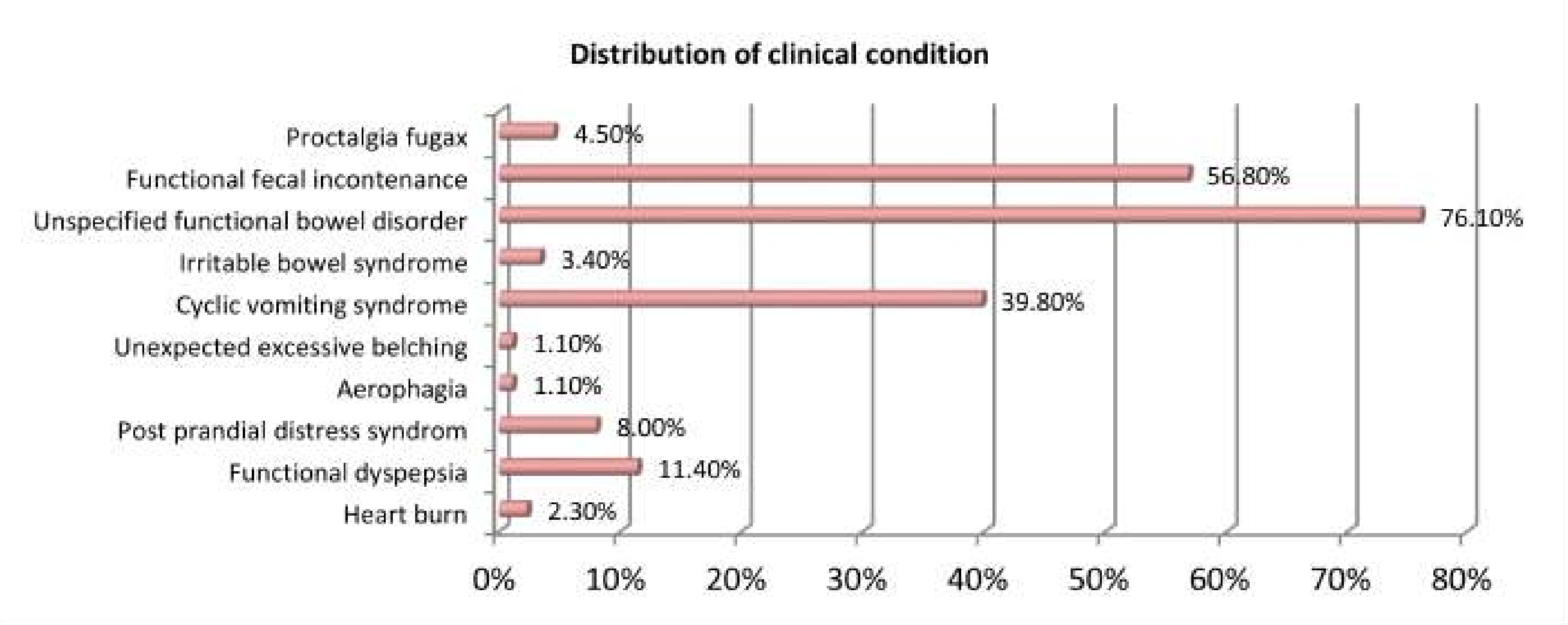

Distribution of gastrointestinal disorders among the Study Subjects $(n=3893)$

Distribution of gastrointestinal disorders is shown in figure 3 . Among the disorders unspecified functional bowel disorder (UFBD) is the most frequent (76.10\%), then functional fecal incontinence (FFI)(56.80\%), cyclic vomiting syndrome(CVS) (39.80\%),functional dyspepsia (FD) $(11.40 \%)$ and post prandial distress (PPD) $(8.00 \%)$ are more frequent respectively.

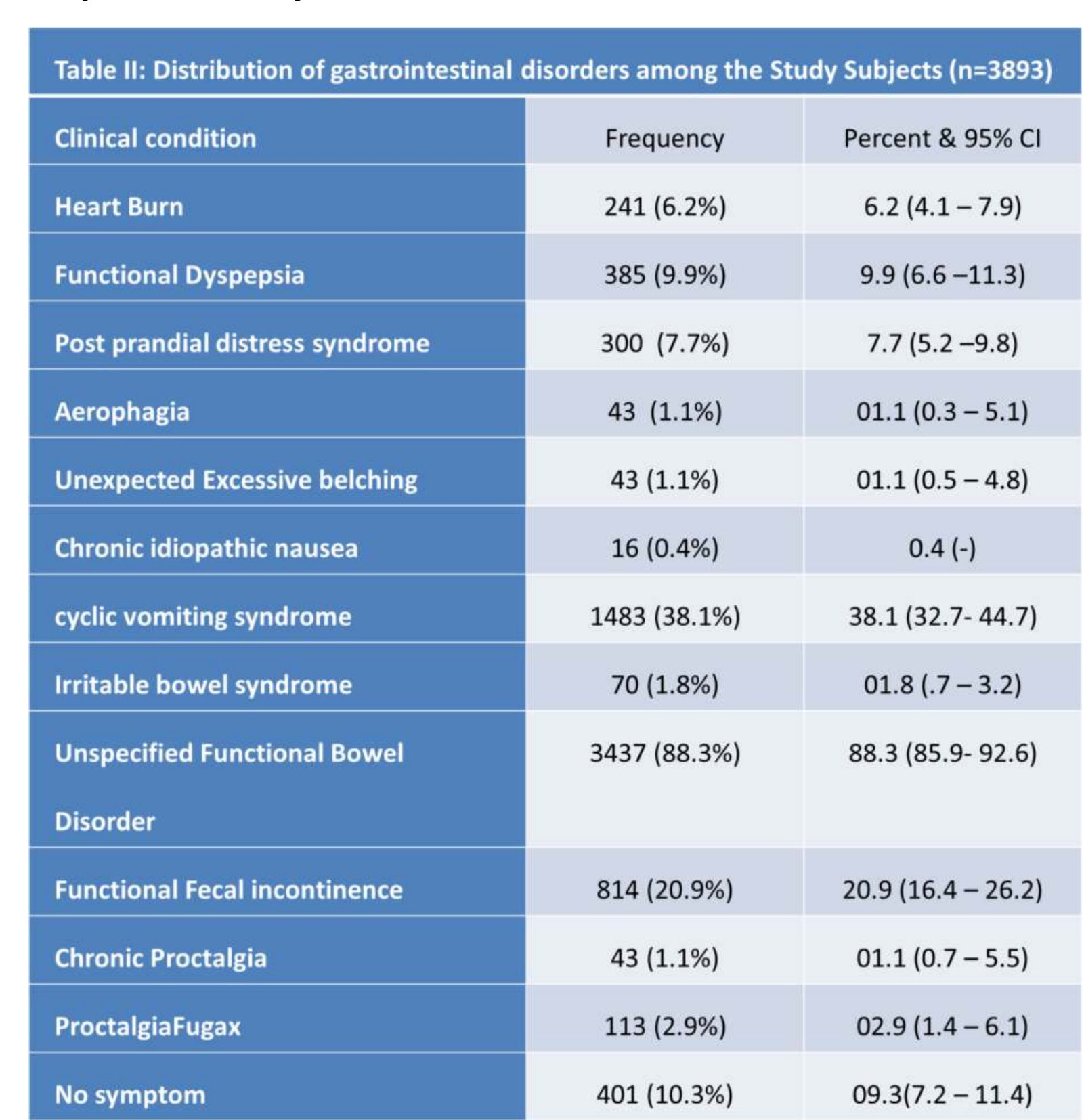

In the study subjects, unspecified functional bowel disorder(UFBD) is the most frequent (76.10\%) symtomp, then functional fecal incontinence (FFI)(56.80\%), cyclic vomiting syndrome(CVS) (39.80\%),functional dyspepsia (FD) (11.40\%) and post prandial distres (PPD) (8.00\%) are more frequent respectively. Among 301 subjects the percentage of no disorder was about $9.3 \%$, single disorder was about $40.9 \%$, double disorders were about $29.6 \%$, Triple disorders were about $11.3 \%$ and more than triple disorders were about $9 \%$.

Comparison of biochemical parameters between GID and NGID groups $(n=91)$

Table 5 compares the average biochemical parameters in diabetic patient with and without GID. None of the parameters were apparently different across groups. performed. $n=$ number of subjects, GID= gastrointestinal disorders, NGID= without gastrointestinal disorders. FSG=Fasting serum glucose; PPG=Postprandial $(2 \mathrm{hrs}$ after breakfast) glucose; STG=Serum Triglyceride; S Chol=Serum total cholesterol; SGPT=Serum glutamate pyruvate transferase; $\mathrm{S}$ creat= Serum creatinine.

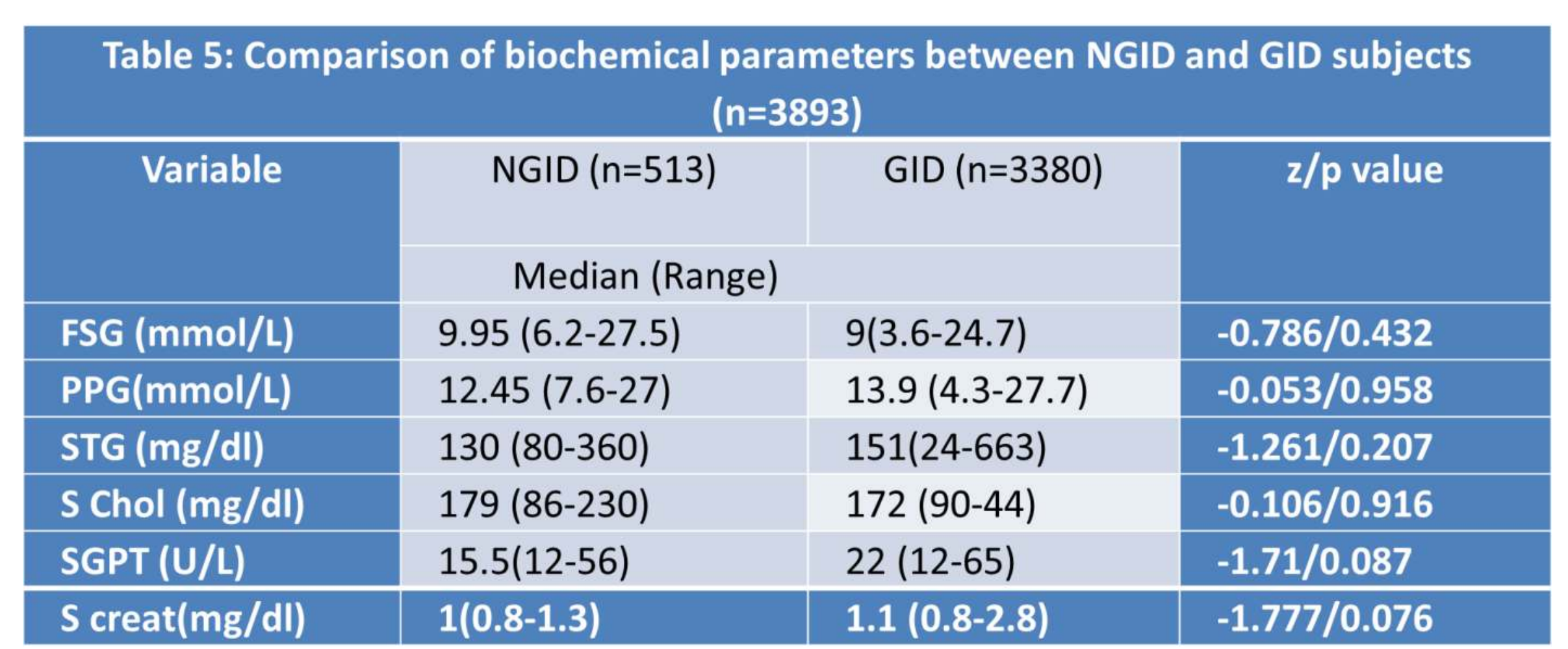

Comparison of Insulinemic status between GID and NGID subjects

Table 6 compares the average Insulinemic parameters in diabetic patients with and without GID. None of the parameters were apparently different across the groups.

Table 6: Comparison of Insulinemic status between GID and NGID ( $n=3893$ )

Subjects Values expressed as median (range). Mann Whitney test was performed. $n=$ number of subjects; $\mathrm{GID}=$ gastrointestinal disorders, $\mathrm{NGID}=$ without gastrointestinal disorders; HOMA-B \%= B cell function assessed by homeostasis model assessment and HOMA-S\%= Insulin sensitivity assessed by homeostasis model assessment.

Multivariate analysis (binary logistic regression) of the association of different disorders of GID with variables of interest

Table 7 shows the multivariate analysis (Binary logistic regression) was done to determine the predictor of GID, GIV, CVS, FFI and UFBD among patients with diabetes adjusted for other variable included in the model. The model is generated considering GID, GIV, CVS, $\mathrm{FFI}$ and UFBD (present or absent) as dependent variable. The model included Sex (Male/female), Residence (Rural/urban), FPG, Serum triglyceride, Serum Total cholesterol, SGPT, Serum Creatinine, $C$ peptide, HOMA B and HOMA S as independent variable. Model summery demonstrates, $R^{2}$ of .323 . Hence $32.3 \%$ of the variation of occurrence of GID, GIV, CVS, FFI and UFBD among the study subjects can be explained by the attributes entered in to the model.

\begin{tabular}{|c|c|c|c|}
\hline \multirow[t]{2}{*}{ Variable } & NGID ( $n=513)$ & GID $(n=3380)$ & \multirow[t]{2}{*}{$z / p$ value } \\
\hline & Median (Range) & & \\
\hline FSG (mmol/L) & $9.95(6.2-27.5)$ & $9(3.6-24.7)$ & $-0.786 / 0.432$ \\
\hline PPG $(\mathrm{mmol} / \mathrm{L})$ & $12.45(7.6-27)$ & $13.9(4.3-27.7)$ & $-0.053 / 0.958$ \\
\hline STG (mg/dl) & $130(80-360)$ & $151(24-663)$ & $-1.261 / 0.207$ \\
\hline $\mathrm{S} \mathrm{Chol} \mathrm{(mg/dl)}$ & $179(86-230)$ & $172(90-44)$ & $-0.106 / 0.916$ \\
\hline SGPT (U/L) & $15.5(12-56)$ & $22(12-65)$ & $-1.71 / 0.087$ \\
\hline$S$ creat $(\mathrm{mg} / \mathrm{dl})$ & $1(0.8-1.3)$ & $1.1(0.8-2.8)$ & $-1.777 / 0.076$ \\
\hline
\end{tabular}

Table 5: Multiple logistic regression analysis of the association of different disorders of GID, GIV, CVS, FFI and UFBD with variables of interest ( $n=91)$.

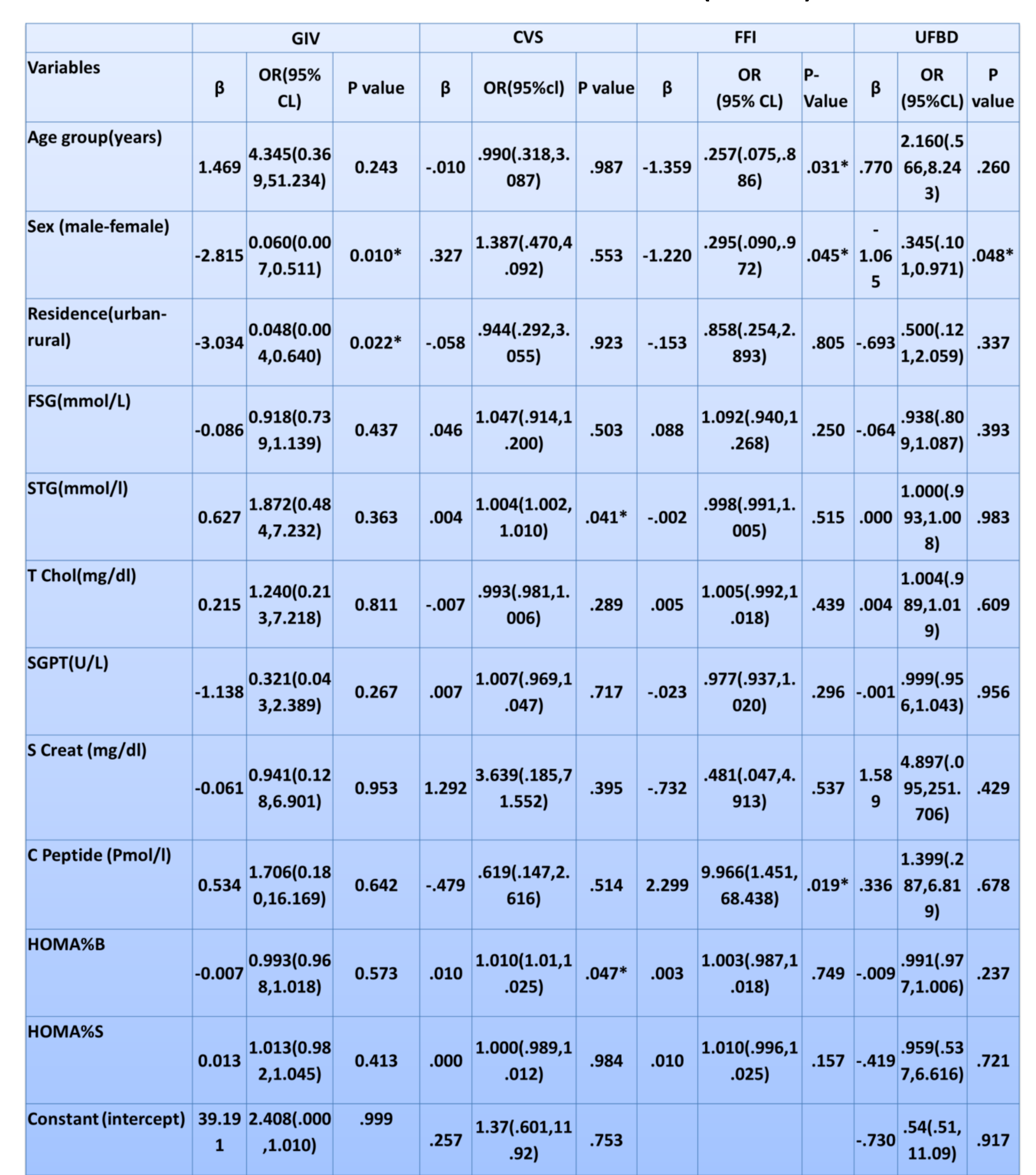

FSG=Fasting serum glucose; PPG=Postprandial ( 2 hrs after breakfast) glucose; $S T G=S e r u m$ Triglyceride; S Chol=Serum total cholesterol; SGPT=Serum glutamate pyruvate transferase; $S$ creat $=$ Serum creatinine; HOMA-B \%= B cell function assessed by homeostasis model assessment and $\mathrm{HOMA}-\mathrm{S} \%=$ Insulin sensitivity assessed by homeostasis model assessment.

Values expressed as Multivariate analysis (Binary logistic regression). Nagelkerke $R^{2} .083$ $\beta=$ standardized regression coefficient; $P<0.05$ was considered as statistically significant; $n$ = number of subjects; UFBD=Unspecified functional bowel disorder; FSG=Fasting serum glucose; PPG=Postprandial ( 2 hrs after breakfast) glucose; STG=Serum Triglyceride; $S$ Chol=Serum total cholesterol; SGPT=Serum glutamate pyruvate transferase; $S$ creat= Serum creatinine. HOMA-B \%= B cell function assessed by homeostasis model assessment and HOMA-S\%= Insulin sensitivity assessed by homeostasis model assessment.

CONCLUSION: Most of the patients with diabetes have one or more types of GI disorders and UFBD is the most frequent disorder. CVS, FFI are the top three GI disorders. G disorders in type 2 DM subjects seem to have an association with male sex and urban residence, Diabetic retinopathy, diabetic nephropathy and presence of IHD. 